\title{
Maternal $V D R$ variants rather than 25-hydroxyvitamin D concentration during early pregnancy are associated with type 1 diabetes in the offspring
}

\author{
Maija E. Miettinen ${ }^{1}$ - Melissa C. Smart ${ }^{2}$ - Leena Kinnunen ${ }^{1} \cdot$ Christopher Mathews $^{2}$. \\ Valma Harjutsalo ${ }^{1,3,4,5}$ - Heljä-Marja Surcel ${ }^{6}$. Christel Lamberg-Allardt $^{7}$. \\ Jakko Tuomilehto ${ }^{1,8,9,10}$ • Graham A. Hitman ${ }^{2}$
}

Received: 21 January 2015 / Accepted: 1 June 2015 /Published online: 26 June 2015

(C) Springer-Verlag Berlin Heidelberg 2015

\begin{abstract}
Aims/hypothesis We investigated whether single nucleotide polymorphisms (SNPs) associated with 25-hydroxyvitamin $\mathrm{D}$ concentration in the metabolic pathway of vitamin D show different genotype distributions between Finnish families with an offspring with type 1 diabetes (cases) and families with a healthy offspring (controls).

Methods A total of 31 SNPs in eight genes were studied in case and control mothers and family members (offspring with type 1 diabetes and healthy siblings, healthy control children and fathers) $(n=2,854)$. The 25 -hydroxyvitamin D concentration was studied in 474 case and 348 matched control mothers during pregnancy.

Results The genotype distributions of 13 SNPs (in the following genes: 7-dehydrocholesterol reductase NADSYN1/ $D H C R 7$, vitamin D receptor $V D R$, group-specific component $G C$ and $C Y P 27 A 1)$ that showed a nominal association with 25 -hydroxyvitamin D concentration $(p<0.05)$ were compared
\end{abstract}

Maija E. Miettinen and Melissa C. Smart are joint first authors of this study.

Jaakko Tuomilehto and Graham A. Hitman are joint senior authors of this study.

Maija E. Miettinen

maija.miettinen@thl.fi

1 National Institute for Health and Welfare, Chronic Disease Prevention Unit, PO Box 30, 00271 Helsinki, Finland

2 Blizard Institute, Barts and the London School of Medicine and Dentistry, Queen Mary University of London, London, UK

3 Folkhälsan Institute of Genetics, Folkhälsan Research Center University of Helsinki, Helsinki, Finland

4 Abdominal Center Nephrology, University of Helsinki and Helsinki University Hospital, Helsinki, Finland between case and control families. SNPs in VDR had different genotype distributions between the case and control mothers (rs1544410, $p=0.007 ;$ rs 731236, $p=0.003$; rs4516035, $p=$ 0.015), two SNPs (rs1544410 and rs731236) remaining significant after correction for multiple testing using a false discovery rate. The mean 25-hydroxyvitamin D concentrations during pregnancy did not differ between the case and control mothers.

Conclusions/interpretation Our preliminary results suggest that the maternal genotypes of SNPs in VDR may influence the in utero environment and thus contribute to the early programming of type 1 diabetes in the fetus. It is possible that the effects are only relevant in the presence of vitamin D insufficiency.

Keywords 25-Hydroxyvitamin D $\cdot$ In utero $\cdot$ Maternal genotype $\cdot$ Pregnancy $\cdot$ Type 1 diabetes $\cdot V D R \cdot$ Vitamin $\mathrm{D}$

5 Research Program Unit, Diabetes and Obesity, University of Helsinki, Helsinki, Finland

6 Impact Assessment Unit, National Institute for Health and Welfare, Oulu, Finland

7 Department of Food and Environmental Sciences, University of Helsinki, Helsinki, Finland

8 Center for Vascular Prevention, Danube-University Krems, Krems, Austria

9 South Ostrobothnia Central Hospital, Seinäjoki, Finland

10 Diabetes Research Group, King Abdulaziz University, Jeddah, Saudi Arabia 


\section{Abbreviations \\ 25OHD 25-Hydroxyvitamin D \\ DBP Vitamin D binding protein \\ FDR False discovery rate \\ SNP Single nucleotide polymorphism \\ VDR Vitamin D receptor}

\section{Introduction}

Vitamin D deficiency, which is most commonly defined as a serum 25-hydroxyvitamin D (25OHD) concentration lower than $50 \mathrm{nmol} / 1$, may increase the risk of autoimmune diseases, diabetes, cancer and cardiovascular diseases [1]. The existing evidence on the association between vitamin $\mathrm{D}$ and type 1 diabetes is inconsistent. Vitamin D supplementation during infancy has been connected with a decreased risk of type 1 diabetes in several [2-4], but not all [5], studies. While it has been suggested that vitamin $\mathrm{D}$ supplementation during pregnancy is associated with a lower risk of type 1 diabetes, and that lower 25OHD concentrations during pregnancy are associated with a higher risk of type 1 diabetes in the offspring [6, $7]$, some studies have not been able to confirm this $[8,9]$. The prevalence of vitamin D deficiency has been shown to be higher in children with multiple pancreatic islet autoantibodies compared with autoantibody-negative children, although the type 1 diabetes did not progress more quickly in the vitamin D-deficient group [10]. In another study, the 25OHD concentration was not associated with islet autoimmunity or the progression of type 1 diabetes in children [11]. Several genes important to the metabolic pathway of vitamin $\mathrm{D}$ have been robustly associated with $25 \mathrm{OHD}$ concentrations, islet autoimmunity or type 1 diabetes [12-17].

The metabolism of vitamin D consists of several hydroxylation reactions catalysed by the members of the cytochrome p450 family. In the skin, vitamin $\mathrm{D}_{3}$ (cholecalciferol) is synthesised from 7-hydrocholesterol. NAD synthetase 1 (NADSYN1) and 7-dehydrocholesterol reductase (DHCR7) together remove 7-hydrocholesterol from the vitamin D pathway. NADSYN1 and DHCR7 genes are located near each other and are often referred to as the NADSYN1/DHCR7 locus.

Vitamin $\mathrm{D}_{3}$ is hydroxylated in the liver to 25OHD (by the cytochrome $\mathrm{p} 450$-containing hydroxylases encoded by CYP2R1 and CYP27A1). Vitamin $\mathrm{D}_{3}$ and 25OHD are transported in the circulation bound to the vitamin $\mathrm{D}$ binding protein (DBP; encoded by the group-specific component $G C$ gene). 25OHD is hydroxylated to the active form of vitamin $\mathrm{D}, 1,25$-dihydroxyvitamin $\mathrm{D}\left(1,25 \mathrm{OHD}_{2}\right)$, in the kidney or target cells (by $1 \alpha$-hydroxylase encoded by $C Y P 27 B 1$ ). Cubilin (encoded by the cubilin gene $C U B N$ ) is needed for the absorption of the 25OHD-DBP complex into the proximal tubules of the kidney. 1,25-dihydroxyvitamin $\mathrm{D}$ is further hydroxylated by 24-hydroxylase (encoded by CYP24A1) to the inactive form of vitamin D [18]. The active form of vitamin D acts through the vitamin $\mathrm{D}$ receptor (encoded by $V D R$ ), which is a nuclear transcription factor that regulates the transcription of vitamin-D sensitive genes. VDRs have been detected in most tissues, and it has been estimated that VDRs can regulate the expression of as many as 500 genes [19].

The aim of the study was to compare the genotype distributions of single nucleotide polymorphisms (SNPs) related to vitamin $\mathrm{D}$ metabolism that showed the strongest association with 25OHD concentration in the Finnish population between case (offspring with type 1 diabetes) and control (non-diabetic offspring) families.

\section{Methods}

Study population A detailed description of the study population of pregnant women (including the eligibility and matching criteria for the case and control mothers) and the serum samples, as well as $25 \mathrm{OHD}$ analysis, has been previously published [8]. Initially, 751 families with offspring with type 1 diabetes and 751 control families around Finland were invited to participate in the study. A saliva sample was collected for DNA extraction from all the participants. A DNA sample was derived from a total of 2,854 individuals (512 case mothers, 470 case fathers, 534 case children, 238 healthy siblings, 379 control mothers, 340 control fathers and 381 control children). Of the 512 case mothers and 379 control mothers for whom a DNA sample was available, a 25OHD concentration was available for 474 and 348 , respectively. The main reasons for missing 25OHD concentrations were that there was no sample, or no sample was left in the Finnish Maternity Cohort sample collection, or that the mother did not give permission to use the stored serum sample for $25 \mathrm{OHD}$ analysis. The mean age at diagnosis of the children with type 1 diabetes was 3.4 years (range $0-7$ years). Written informed consent was collected from all participants. The ethics committee of the Hospital District of Helsinki and Uusimaa approved the study.

Selection and genotyping of SNPs DNA was isolated using Oragene kits (DNA Genotek, Ottawa, ON, Canada) from 2,854 individuals who submitted saliva samples. A total of 31 SNPs in eight candidate genes were genotyped using TaqMan (Applied Biosystems, Paisley, UK): VDR (rs731236, rs1544410, rs7975232, rs2228570, rs4516035, rs10783219); GC (rs4588, rs7041, rs12512631, rs2282679, rs3755967, rs17467825, rs2298850); CYP2Rl (rs10741657, rs2060793, rs1993116, rs7116978, rs12794714, rs10500804); CYP27B1 (rs108770112, rs4646536); CYP24A1 (rs6013897); CYP27A1 (rs17470271); CUBN (rs3740165); and NADSYN1/ DHCR7 (rs12785878, rs3829251, rs7944926, rs12800438, rs3794060, rs4945008, rs4944957). All the SNPs were in 
genes associated with the vitamin D pathway and have previously shown an association with either serum 25OHD concentration or type 1 diabetes [16, 17, 20-27].

Statistical methods All the statistical analyses were performed using Intercooled Stata 10 for Windows (Stata Statistical Software: Release 10, 2007; StataCorp, College Station, TX, USA). Linear regression was used to analyse the association between 25OHD concentrations and SNPs related to vitamin D metabolism. Due to the known seasonal variation of 25OHD concentrations in Finland, the analyses were adjusted for the month of sample collection. Pearson's $\chi^{2}$ test was used for differences in the proportions of the case and control mothers by genotype. The sample size was derived from power calculations from our original paper [8]. When comparing the distribution of SNP genotypes, multiple testing was controlled for using the false discovery rate (FDR) method (a step-up procedure described by Benjamini and Hochberg [28], using 0.05 as the criterion). If the original $p$ value was smaller than the Benjamini-Hochberg critical value, the difference in genotype distribution was considered statistically significant.

\section{Results}

Genotyping All the SNPs were in Hardy-Weinberg equilibrium with the exception of $V D R$ SNP rs7975232, which was removed from further analyses. Around $95-99 \%$ of the samples were successfully genotyped, and $100 \%$ of genotypes were concordant in 120 duplicate samples.

From the 31 SNPs, we selected those that had the strongest association with $25 \mathrm{OHD}$ (with a nominal $p$ value of $<0.05$ ) in the Finnish population (13 SNPs in four genes, NADSYN1/ DHCR7, VDR, GC and CYP27A; Table 1), and then compared the genotype frequencies between the case and control families (Table 2). Three SNPs demonstrated different genotype distributions between the mothers of children with type 1 diabetes and the control mothers; all these were localised to $V D R$ (rs1544410, $p=0.007$; rs731236, $p=0.003$; and rs4516035, $p=0.015$ ). Two SNPs (rs1544410 and rs731236) remained statistically significant after correction for multiple testing with FDR. The difference in the proportion of minor alleles between the case and control mothers was not statistically significant (Table 2).

No significant differences were observed between the allele frequencies of the 13 studied SNPs in the fathers, between the case and control children, or between the case children and their healthy siblings. Similarly, in the case families, no allele of the 13 SNPs studied was preferentially transmitted to the child with type 1 diabetes.

Maternal serum 25OHD concentrations The mean 25OHD concentrations in the case (44.9 nmol/1, $n=474)$ and control (43.7 nmol/1, $n=348)$ mothers did not differ significantly when adjusted for the month of sample collection. In total, $69.1 \%$ of all the mothers showed vitamin D deficiency ( $25 \mathrm{OHD}$ concentration $<50 \mathrm{nmol} / \mathrm{l}$ ). Only $3.7 \%$ of the case

Table 1 Association of mother's genotype with 25OHD concentration

\begin{tabular}{|c|c|c|c|c|c|c|c|c|c|c|c|c|}
\hline \multirow[t]{3}{*}{ SNP } & \multirow[t]{3}{*}{ Gene } & \multicolumn{9}{|c|}{$25 \mathrm{OHD}$ concentration $(\mathrm{nmol} / \mathrm{l})^{\mathrm{a}}$ by genotype } & \multirow{3}{*}{$\begin{array}{l}p \text { - } \\
\text { values }\end{array}$} & \multirow[t]{3}{*}{$n$} \\
\hline & & \multicolumn{3}{|l|}{11} & \multicolumn{3}{|l|}{12} & \multicolumn{3}{|l|}{22} & & \\
\hline & & Mean & LCI & UCI & Mean & LCI & UCI & Mean & LCI & UCI & & \\
\hline rs4945008 & NADSYN1/DHCR7 & 42.88 & 41.18 & 44.66 & 41.07 & 39.97 & 42.19 & 39.33 & 37.28 & 41.48 & 0.029 & 766 \\
\hline rs 12785878 & NADSYN1/DHCR7 & 42.94 & 41.25 & 44.69 & 41.03 & 39.95 & 42.14 & 39.21 & 37.19 & 41.34 & 0.021 & 768 \\
\hline rs4944957 & NADSYN1/DHCR7 & 42.69 & 40.95 & 44.51 & 41.06 & 39.93 & 42.22 & 38.70 & 37.38 & 41.71 & 0.055 & 728 \\
\hline rs7944926 & NADSYN1/DHCR7 & 42.97 & 41.24 & 44.78 & 41.04 & 39.94 & 42.17 & 39.20 & 37.16 & 41.35 & 0.022 & 757 \\
\hline rs 3794060 & NADSYN1/DHCR7 & 42.74 & 41.01 & 44.54 & 40.70 & 39.59 & 41.84 & 38.77 & 36.71 & 40.95 & 0.016 & 733 \\
\hline rs 12800438 & NADSYN1/DHCR7 & 42.98 & 41.29 & 44.74 & 41.12 & 40.03 & 42.23 & 39.34 & 37.31 & 41.47 & 0.024 & 775 \\
\hline rs4516035 & $V D R$ Prom & 43.03 & 41.22 & 44.92 & 41.23 & 40.17 & 42.32 & 39.50 & 37.69 & 41.40 & 0.022 & 764 \\
\hline rs731236 & $V D R$ Taq 1 & 40.29 & 38.86 & 41.78 & 42.12 & 40.91 & 43.36 & 44.02 & 41.54 & 46.66 & 0.025 & 769 \\
\hline rs 1544410 & $V D R$ Bsm1 & 40.25 & 38.81 & 41.75 & 42.01 & 40.78 & 43.27 & 43.84 & 41.33 & 46.51 & 0.033 & 747 \\
\hline rs10783219 & $V D R$ & 39.93 & 38.43 & 41.48 & 41.75 & 40.60 & 42.92 & 43.64 & 41.32 & 46.10 & 0.022 & 751 \\
\hline rs12512631 & $G C$ & 40.23 & 38.72 & 41.80 & 41.94 & 40.79 & 43.12 & 43.71 & 41.43 & 46.11 & 0.029 & 755 \\
\hline rs 4588 & $G C ; \mathrm{T} 436 \mathrm{~K}$ & 42.10 & 40.72 & 43.52 & 40.23 & 38.78 & 41.72 & 38.44 & 35.64 & 41.45 & 0.050 & 737 \\
\hline rs 17470271 & CYP27A1 & 42.04 & 40.71 & 43.42 & 40.59 & 39.15 & 42.09 & 39.19 & 36.38 & 42.22 & 0.030 & 750 \\
\hline
\end{tabular}

${ }^{\text {a }}$ Adjusted for the month the serum sample was taken

11, common homozygotes; 12, heterozygotes; 22, rare homozygotes; LCI, lower confidence interval; UCI, upper confidence interval 
Table 2 Genotype distribution of three VDR SNPs in case and control mothers

\begin{tabular}{|c|c|c|c|c|c|}
\hline SNP & Case mothers & Control mothers & OR $(95 \% \mathrm{CI})$ & $p$ values & $\begin{array}{l}\text { Benjamini-Hochberg } \\
\text { critical value }^{\mathrm{a}}\end{array}$ \\
\hline rs 1544410 & & & & $0.007^{\mathrm{b}}$ & 0.008 \\
\hline \multicolumn{6}{|c|}{ Genotypes, $n(\%)$} \\
\hline AA & $48(10.2)$ & $42(12.0)$ & $0.85(0.58,1.26)$ & 0.41 & \\
\hline $\mathrm{AG}$ & $227(48.1)$ & $130(37.0)$ & $1.30(1.10,1.53)$ & 0.0016 & \\
\hline GG & $197(41.7)$ & $179(51.0)$ & $0.81(0.70,0.94)$ & 0.0062 & \\
\hline \multicolumn{6}{|c|}{ Alleles, $n(\%)$} \\
\hline A & $323(34.2)$ & $214(30.5)$ & $1.19(0.96,1.46)$ & 0.11 & \\
\hline G & $621(65.8)$ & $488(69.5)$ & & & \\
\hline rs 731236 & & & & $0.003^{\mathrm{b}}$ & 0.004 \\
\hline \multicolumn{6}{|c|}{ Genotypes, $n(\%)$} \\
\hline $\mathrm{CC}$ & $49(9.9)$ & $43(12.3)$ & $0.80(0.55,1.18)$ & 0.26 & \\
\hline $\mathrm{CT}$ & $243(49.0)$ & $130(37.2)$ & $1.32(1.12,1.55)$ & 0.0007 & \\
\hline TT & $204(41.1)$ & $176(50.4)$ & $0.82(0.70,0.95)$ & 0.0075 & \\
\hline \multicolumn{6}{|c|}{ Alleles, $n(\%)$} \\
\hline $\mathrm{C}$ & $341(34.4)$ & $216(30.9)$ & $1.17(0.95,1.44)$ & 0.14 & \\
\hline $\mathrm{T}$ & $651(65.6)$ & $482(69.1)$ & & & \\
\hline rs 4516035 & & & & 0.015 & 0.012 \\
\hline \multicolumn{6}{|c|}{ Genotypes, $n(\%)$} \\
\hline $\mathrm{CC}$ & $124(25.1)$ & $63(18.4)$ & $1.37(1.04,1.79)$ & 0.0215 & \\
\hline $\mathrm{CT}$ & $225(45.5)$ & $189(55.1)$ & $0.83(0.72,0.95)$ & 0.0066 & \\
\hline TT & $145(29.4)$ & $91(26.5)$ & $1.11(0.89,1.38)$ & 0.37 & \\
\hline \multicolumn{6}{|c|}{ Alleles, $n(\%)$} \\
\hline $\mathrm{C}$ & 473 (47.9) & $315(45.9)$ & $1.08(0.89,1.32)$ & 0.43 & \\
\hline $\mathrm{T}$ & $515(52.1)$ & $371(54.1)$ & & & \\
\hline
\end{tabular}

${ }^{\text {a }}$ Multiple testing correction with the FDR approach (Benjamini-Hochberg step-up procedure). If the original $p$ value is less than the BenjaminiHochberg critical value, it is considered statistically significant

${ }^{\mathrm{b}}$ Statistically significant after multiple testing correction using the FDR approach

mothers and $1.7 \%$ of the control mothers had an optimal vitamin D status $(>75 \mathrm{nmol} / \mathrm{l})$ in the first trimester of pregnancy. The 25OHD concentrations changed almost twofold by season $(34.3 \pm 11.0 \mathrm{nmol} / \mathrm{l}$ in March, $61.2 \pm 20.8 \mathrm{nmol} / 1$ in August; Fig. 1).

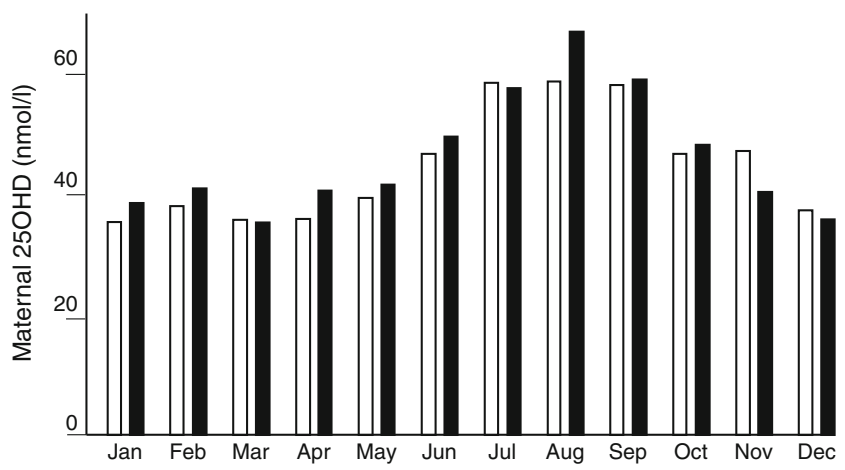

Fig. 1 Maternal 25OHD concentrations by case (black bars) and control (white bars) status and month of sampling

\section{Discussion}

In this study, we investigated the genotype distributions of SNPs related to vitamin D metabolism between families whose offspring had type 1 diabetes (cases) and families with healthy offspring (controls). Our results suggest that certain maternal $V D R$ variants are associated with a child's risk of type 1 diabetes independent of the child's genotype, and may thus influence the in utero environment and contribute to the early programming of type 1 diabetes in the fetus.

In our previously published study [8], we did not find a difference in $25 \mathrm{OHD}$ concentrations in pregnant women in the first trimester who gave birth to a child with type 1 diabetes when they were strictly matched with control families with no diabetic children, or in 25OHD concentrations during pregnancies that produced children with type 1 diabetes vs their healthy siblings. This is in contrast to a smaller study of Norwegian women [7] in which low 25OHD concentrations (mainly in the last trimester) were associated with an increased risk of type 1 diabetes. However, more than $70 \%$ of the 
Norwegian women had a $25 \mathrm{OHD}$ level $>50 \mathrm{nmol} / \mathrm{l}$, compared with $31 \%$ of Finnish women.

At this point, we can only speculate on the possible biological mechanisms that seem to connect certain maternal $V D R$ variants with risk of type 1 diabetes in a child. The 13 SNPs that we used to compare the genotype frequencies between case and control mothers were selected on the basis of a nominal association with 25OHD concentration. However, based on the data provided in the present study, there is no reason to assume that the connection of the $V D R$ variants with maternal 25OHD concentration would necessarily explain the increase in risk of type 1 diabetes in the children of mothers carrying these $V D R$ variants. The association of the VDR variants with $25 \mathrm{OHD}$ concentration can rather be seen as a marker of an (unknown) effect of the variants on maternal vitamin D metabolism and/or VDR function that further associate with the risk of type 1 diabetes in the child.

The vitamin D receptor is a transcription factor with hundreds of target genes [19]. We have previously reported that a genetic variation in $V D R$ is a determinant of its expression [29]. It is thus possible that the genetic variation in maternal $V D R$ modifies the genetic effects of vitamin $\mathrm{D}$, some of which, possibly together with vitamin $\mathrm{D}$ deficiency, may contribute to the autoimmune process in the developing fetus and thus to the pathogenesis of type 1 diabetes. This would be consistent with the Diabetes Autoimmunity Study in the Young (DAISY), which demonstrated an interaction between VDR and protein tyrosine phosphatase, non-receptor type 2 gene (PTPN2), affecting the risk of progression to type 1 diabetes [13]. The fact that no difference in the genotype distribution of SNPs of VDR was seen in the present study between children with type 1 diabetes and control children suggests that the effect is associated with the maternal in utero environment and developmental processes during pregnancy.

$V D R$ is expressed in placenta and its expression levels rise in late pregnancy compared with mid-pregnancy [30]. Since the genomic actions of ligand binding to the VDR include modulation of the immune system with a shift to a $\mathrm{T}$ helper type 2 cytokine response pattern [31], the genomic actions of maternal VDR may assume a greater role in the presence of a reduced supply of 25OHD to the fetus determined by both environment and genotype.

The focus of genetic studies of type 1 diabetes has been the affected child, and the effect of the maternal genotype independent of the child's genotype has not previously been described as a determinant of the child's risk of type 1 diabetes. However, the maternal genotype has previously been shown to be associated with, for example, the child's brain morphology [32], cognitive development [33], risk of autism [34] and atopic dermatitis [35]. The fact that type 1 diabetes-associated autoantibodies that precede the diagnosis of type 1 diabetes can appear only months after birth [36] suggests a fetal programming of the disease.
The clinical significance of our findings is not clear. Several genes contribute jointly to the development of type 1 diabetes, and thus a certain genetic marker alone may have only a minor impact on susceptibility to the disease. Our results require independent validation in a larger, wellcharacterised dataset of cases (the mothers of children with early-onset type 1 diabetes) and well-matched controls, and in relatively homogenous populations, for whom 25OHD levels could be recorded and genotype data were available. It is also important to investigate whether the genetic variation of VDR affects 25OHD concentrations in the fetus by, for example, collecting cord blood samples. Our results highlight the importance of investigating factors that affect the in utero environment and may thus contribute to the early programming of type 1 diabetes.

Although the present study had adequate power to detect the effect of certain maternal SNPs of VDR on the risk of type 1 diabetes in the child, the effect of other SNPs may not be seen in the present study due to its limited power. If our results can be validated, this will be the first time that the interaction between maternal genes and environment will have been demonstrated to influence autoimmunity in utero, which, together with a child's high-risk type 1 diabetes-associated genotype, determines the susceptibility to type 1 diabetes. The results of the present study emphasise that the in utero environment should be a line of investigation when trying to find methods for the primary prevention of type 1 diabetes.

Funding This study was supported by the Finnish Academy (grant 127219), the European Foundation for the Study of Diabetes, the Novo Nordisk Foundation, the Diabetes Research Foundation, the EVO funding of the South Ostrobothnia Central Hospital from the Ministry of Health and Social Affairs (EVO1107), the Biomedicum Helsinki Foundation, the Jalmari and Rauha Ahokas Foundation, the Yrjö Jahnsson Foundation, the Suoma Loimaranta-Airila Fund, the Onni and Hilja Tuovinen Foundation and the Juho Vainio Foundation.

Duality of interest The authors declare that there is no duality of interest associated with this manuscript. The sponsors had no role in the design, data collection, data analysis, data interpretation, writing or revision of the report.

Contribution statement GAH and JT had full access to all the data in the study and take responsibility for the integrity of the data and the accuracy of the data analysis. GAH, JT, CL-A and VH are responsible for the study's concept and design. MEM, LK, H-MS, MCS (genotyping) and JT acquired the data. MCS, MEM, GAH, JT, CM and VH are responsible for the analysis and interpretation of the data. MCS, MEM, GAH and JT drafted the manuscript. LK, CM, H-MS, CL-A, VH undertook the critical revision of the manuscript for important intellectual content. MCS, CM and VH undertook the statistical analysis. CM contributed administrative, technical and material support. GAH and JT supervised the study. All authors gave their final approval of the version to be published. 


\section{References}

1. Holick MF (2007) Vitamin D deficiency. N Engl J Med 357: 266-281

2. The EURODIAB Substudy 2 Study Group (1999) Vitamin D supplement in early childhood and risk for type I (insulin-dependent) diabetes mellitus. Diabetologia 42:51-54

3. Hyppönen E, Läärä E, Reunanen A, Järvelin MR, Virtanen SM (2001) Intake of vitamin D and risk of type 1 diabetes: a birthcohort study. Lancet 358:1500-1503

4. Stene LC, Joner G, Norwegian Childhood Diabetes Study Group (2003) Use of cod liver oil during the first year of life is associated with lower risk of childhood-onset type 1 diabetes: a large, population-based, case-control study. Am J Clin Nutr 78(6):1128-1134

5. Brekke HK, Ludvigsson J (2007) Vitamin D supplementation and diabetes-related autoimmunity in the ABIS study. Pediatr Diabetes $8(1): 11-14$

6. Stene LC, Ulriksen J, Magnus P, Joner G (2000) Use of cod-liver oil during pregnancy associated with lower risk of type 1 diabetes in the offspring. Diabetologia 43:1093-1098

7. Sørensen IM, Joner G, Jenum PA, Eskild A, Torjesen PA, Stene LC (2012) Maternal serum levels of 25-hydroxy-vitamin D during pregnancy and risk of type 1 diabetes in the offspring. Diabetes 61:175-178

8. Miettinen ME, Reinert L, Kinnunen L et al (2012) Serum 25hydroxyvitamin $\mathrm{D}$ level during early pregnancy and type 1 diabetes risk in the offspring. Diabetologia 55:1291-1294

9. Marjamäki L, Niinistö S, Kenward MG et al (2010) Maternal intake of vitamin D during pregnancy and risk of advanced beta cell autoimmunity and type 1 diabetes in offspring. Diabetologia 53: $1599-1607$

10. Raab J, Giannopoulou EZ, Schneider S et al (2014) Prevalence of vitamin D deficiency in pre-type 1 diabetes and its association with disease progression. Diabetologia 57:902-908

11. Simpson M, Brady H, Yin X et al (2011) No association of vitamin $\mathrm{D}$ intake or 25-hydroxyvitamin D levels in childhood with risk of islet autoimmunity and type 1 diabetes: the Diabetes Autoimmunity Study in the Young (DAISY). Diabetologia 54:2779-2788

12. Orton SM, Morris AP, Herrera BM et al (2008) Evidence for genetic regulation of vitamin D status in twins with multiple sclerosis. Am J Clin Nutr 88:441-447

13. Frederiksen B, Liu E, Romanos J et al (2013) Investigation of the vitamin $\mathrm{D}$ receptor gene (VDR) and its interaction with protein tyrosine phosphatase, non-receptor type 2 gene (PTPN2) on risk of islet autoimmunity and type 1 diabetes: the Diabetes Autoimmunity Study in the Young (DAISY). J Steroid Biochem Mol Biol 133:51-57

14. Frederiksen BN, Kroehl M, Fingerlin TE et al (2013) Association between vitamin $\mathrm{D}$ metabolism gene polymorphisms and risk of islet autoimmunity and progression to type 1 diabetes: the diabetes autoimmunity study in the young (DAISY). J Clin Endocrinol Metab 98:E1845-E185

15. Barry EL, Rees JR, Peacock JL et al (2014) Genetic variants in CYP2R1, CYP24A1, and VDR modify the efficacy of vitamin D3 supplementation for increasing serum 25-hydroxyvitamin D levels in a randomized controlled trial. J Clin Endocrinol Metab 99:E2133-E2137

16. Cooper JD, Smyth DJ, Walker NM et al (2011) Inherited variation in vitamin $\mathrm{D}$ genes is associated with predisposition to autoimmune disease type 1 diabetes. Diabetes 60:1624-1631

17. Wang TJ, Zhang F, Richards JB et al (2010) Common genetic determinants of vitamin D insufficiency: a genome-wide association study. Lancet 376:180-188
18. Saccone D, Asani F, Bornman L (2015) Regulation of the vitamin $\mathrm{D}$ receptor gene by environment, genetics and epigenetics. Gene 561:171-180

19. Norman AW (2008) From vitamin D to hormone D: fundamentals of the vitamin D endocrine system essential for good health. Am J Clin Nutr 88:491S-499S

20. Uitterlinden AG, Fang Y, Van Meurs JBJ, Pols HA, Van Leeuwen JPTM (2004) Genetics and biology of vitamin D receptor polymorphisms. Gene 338:143-156

21. Nejentsev S, Cooper JD, Godfrey L et al (2004) Analysis of the vitamin $\mathrm{D}$ receptor gene sequence variants in type 1 diabetes. Diabetes 53:2709-2712

22. Bailey R, Cooper JD, Zeitels L et al (2007) Association of the vitamin D metabolism gene CYP27B1 with type 1 diabetes. Diabetes 56:2616-2621

23. Ramos-Lopez E, Brück P, Jansen T, Herwig J, Badenhoop K (2007) CYP2R1 (vitamin D 25-hydroxylase) gene is associated with susceptibility to type 1 diabetes and vitamin D levels in Germans. Diabetes Metab Res Rev 23:631-636

24. Ahn J, Albanes D, Berndt SI et al (2009) Vitamin D-related genes, serum vitamin D concentrations and prostate cancer risk. Carcinogenesis 30:769-776

25. Ahn J, Yu K, Stolzenberg-Solomon R et al (2010) Genome-wide association study of circulating vitamin D levels. Hum Mol Genet 19:2739-2745

26. McGrath JJ, Saha S, Burne THJ, Eyles DW (2010) A systematic review of the association between common single nucleotide polymorphisms and 25-hydroxyvitamin D concentrations. J Steroid Biochem Mol Biol 121:471-477

27. Ramos-Lopez E, Lange B, Penna-Martinez M et al (2010) The role of cubilin gene polymorphisms and their influence on 25OHD3 and $1,25(\mathrm{OH}) 2 \mathrm{D} 3$ plasma levels in type 1 diabetes patients. J Steroid Biochem Mol Biol 121:442-444

28. Benjamini Y, Hochberg Y (1995) Controlling the false discovery rate - a practical and powerful approach to multiple testing. J R Stat Soc B57:289-300

29. Ogunkolade B-W, Boucher BJ, Prahl JM et al (2002) Vitamin D receptor (VDR) mRNA and VDR protein levels in relation to vitamin D status, insulin secretory capacity, and VDR genotype in Bangladeshi Asians. Diabetes 51:2294-2300

30. Shahbazi M, Jeddi-Tehrani M, Zareie M et al (2011) Expression profiling of vitamin $\mathrm{D}$ receptor in placenta, decidua and ovary of pregnant mice. Placenta 32:657-664

31. Guillot X, Semerano L, Saidenberg-Kermanac'h N, Falgarone G, Boissier MC (2010) Vitamin D and inflammation. Joint Bone Spine 77:552-557

32. van der Knaap NJ, El Marroun H, Klumpers F et al (2014) Beyond classical inheritance: the influence of maternal genotype upon child's brain morphology and behavior. J Neurosci 16(34):95169521

33. Pilsner JR, Hu H, Wright RO et al (2010) Maternal MTHFR genotype and haplotype predict deficits in early cognitive development in a lead-exposed birth cohort in Mexico City. Am J Clin Nutr 92: 226-234

34. James SJ, Melnyk S, Jernigan S et al (2010) A functional polymorphism in the reduced folate carrier gene and DNA hypomethylation in mothers of children with autism. Am J Med Genet B Neuropsychiatr Genet 153B:1209-1220

35. Esparza-Gordillo J, Matanovic A, Marenholz I et al (2015) Maternal filaggrin mutations increase the risk of atopic dermatitis in children: an effect independent of mutation inheritance. PLoS Genet 11: e1005076

36. Siljander HT, Simell S, Hekkala A et al (2009) Predictive characteristics of diabetes-associated autoantibodies among children with HLA-conferred disease susceptibility in the general population. Diabetes 58:2835-2842 